\title{
Indole bearing thiadiazole analogs: synthesis, $\beta$-glucuronidase inhibition and molecular docking study
}

\author{
Noor Barak Almandil ${ }^{1 \dagger}$, Muhammad Taha ${ }^{1 *+}$ (D) , Mohammed Gollapalli ${ }^{3 \dagger}$, Fazal Rahim ${ }^{2 \dagger}$, Mohamed Ibrahim ${ }^{1 \dagger}$, \\ Ashik Mosaddik ${ }^{1 \dagger}$ and El Hassane Anouar ${ }^{4+}$
}

\begin{abstract}
Background: Indole based thiadiazole derivatives (1-22) have synthesized, characterized by NMR and HREI-MS and evaluated for $\beta$-Glucuronidase inhibition. All compounds showed outstanding $\beta$-glucuronidase activity with $I_{50}$ values ranging between $0.5 \pm 0.08$ to $38.9 \pm 0.8 \mu \mathrm{M}$ when compared with standard D-saccharic acid 1,4 lactone $\left(\mathrm{IC}_{50}\right.$ value of $48.1 \pm 1.2 \mu \mathrm{M}$ ). The compound $\mathbf{6}$, a 2,3-dihydroxy analog was found the most potent among the series with $I_{50}$ value $0.5 \pm 0.08 \mu \mathrm{M}$. Structure activity relationship has been established for all compounds. To confirm the binding interactions of these newly synthesized compounds, molecular docking study have been carried out which reveal that these compounds established stronger hydrogen bonding networks with active site residues.
\end{abstract}

Keywords: Synthesis, Indole, Thiadiazole, $\beta$-Glucuronidases, Molecular docking, SAR

\section{Background}

$\beta$-Glucuronidases enzymes belong to family glycoside hydrolase GH1, GH2 and GH79, and reduce glucuronic acid sugar moiety from non-reducing termini. It has been used in disease diagnosis, gene manipulation and food industry. Recently it has drawn more attention to enhance the efficacy by modifying natural glucuronides [1-5]. $\beta$-Glucuronidase present in microsomes and lysosomes and eliminate from body through urinary track [6]. Activity of $\beta$-glucuronidase increases in many diseases like AIDS, inflammation, cancer and hepatic disease [7]. Cholelithiasis is originated in human bile due to endogenous biliary $\beta$-glucuronidase which is related with deconjugation of bilirubin glucuronidase [8]. Increase level of this enzyme is associated with some urinary disorder like active pyelonephritis, cancer of kidney bladder

\footnotetext{
*Correspondence: taha_hej@yahoo.com; mtaha@iau.edu.sa ${ }^{\dagger}$ Noor Barak Almandil, Muhammad TahaMohammed Gollapalli, Fazal Rahim, Mohamed Ibrahim, Ashik Mosaddik, and El Hassane Anouar have equal contribution

${ }^{1}$ Department of Clinical Pharmacy, Institute for Research and Medical Consultations (IRMC), Imam Abdulrahman Bin Faisal University, P. O. Box 1982, Dammam 31441, Saudi Arabia

Full list of author information is available at the end of the article
}

and acute renal necrosis [9]. $\beta$-Glucuronidase in humane has resulted in mucopolysaccharidosis type VII (MPS VII; Sly syndrome), which is characterized by growth of glycosaminoglycans in cells of most tissues $[10,11]$. Indole is an important class of compounds with wide range of application in medicinal chemistry [12, 13]. Variety of compounds having indole is the basic unit possesses antitumor applications $[14,15]$. Many compounds containing indole scaffold have effect in many physiological processes. Indole with 5-HT receptor activity as agonist and antagonist is the most recent synthetic interest in medicinal chemistry $[16,17]$.

The thiadiazole skeleton constitutes an important central template for a wide variety of biologically active compounds, having many pharmacological applications 2-amino-1,3,4-thiadiazole and certain structurally related compounds have been known for 50 years to have antitumor activity [18]. Compounds of this class are uricogenic agents in man [19]. Both the antitumor and the uricogenic activities can be prevented or reversed by nicotinamide [20, 21]. Variety of thiadiazole derivatives possess interesting biological activities and are of great interest to chemist [22]. Many bioactive molecules in the field of drugs and pharmaceuticals contained thiadiazole moiety 
[23-25]. Thiadiazole derivatives biological applications include antibacterial, anticonvulsant, anti-leishmanial, anticancer, antidepressant, anti-inflammatory, antioxidant and anti-tuberculosis [26-34]. The indole and related heterocyclic compounds have also great importance in the field of chemistry [35].

Keeping in view the great biological potential of indole and thiadiazole analogs here in this study we have planned to synthesize the hybrid molecules of indole based thiadiazole derivatives with the hope that it may showed greater potential. After evaluation for biological potential we have found outstanding results which support our previous hypothesis. Here we are reporting synthesis of indole based thiadiazole derivatives, its characterization, $\beta$-glucuronidase inhibition and molecular docking studies.

\section{Result and discussion Chemistry}

A series of indole based thiadiazole were synthesized by refluxing ethyl $1 \mathrm{H}$-indole-5-carboxylate (a) with hydrazine hydrate in ethanol for $2 \mathrm{~h}$ to afford $1 H$-indole5 -carbohydrazide (b) refluxed with Lawesson's reagent in toluene yielded corresponding thio-analogue (c). Thiohydrazide (c) was then treated with various aryl aldehydes to form cyclized adducts 1-22 (Table 1) in the presence of $\mathrm{POCl}_{3}$ (Scheme 1). Upon completion of reaction (monitored with TLC), product was recrystallized from methanol and purified by washing. Spectral data including ${ }^{1} \mathrm{H}$-NMR, ${ }^{13} \mathrm{C}$-NMR, and HREI-MS for all synthesized compounds were recorded.

\section{Biological activity}

In the continuation of our effort for enzyme inhibition [36], we have synthesized series of indole based thiadiazole derivatives, a new class of $\beta$-glucuronidase inhibitors. All the compounds (1-22) were screened for $\beta$-glucuronidase activity. All the compounds showed outstanding inhibition when compared with standard D-saccharic acid 1,4 lactone with $\mathrm{IC}_{50}$ value $48.1 \pm 1.2 \mu \mathrm{M}$ (Table 1). Structure activity relationship (SAR) has been established mainly based on the substitution pattern on phenyl ring attached to thiadiazole. The compound 6, a 2,3-dihydroxy analog was found the most potent among the series with $\mathrm{IC}_{50}$ value $0.5 \pm 0.08 \mu \mathrm{M}$. The greater potential shown by this compound is seems mainly due to the hydroxyl group on phenyl ring which might be involve in hydrogen bonding with the active site of enzyme. If we compare analog 6 with other dihydroxy substituted analogs like 5, a 2,5-dihydroxy analog $\left(\mathrm{IC}_{50}=1.82 \pm 0.01 \mu \mathrm{M}\right)$, 7, a 3,4-dihydroxy analog $\left(\mathrm{IC}_{50}=1.1 \pm 0.08 \mu \mathrm{M}\right)$, and 8, a 2,4-dihydroxy analog $\left(\mathrm{IC}_{50}=2.30 \pm 0.1 \mu \mathrm{M}\right)$, the analog 6 is superior. The slight difference in the potential of these analogs is seems due to the difference in position of substituent. The mono-hydroxy analogs like 3, a 2-hydroxy analog $\left(\mathrm{IC}_{50}=3.1 \pm 0.01 \mu \mathrm{M}\right)$, 4, a 3-hydroxy analog $\left(\mathrm{IC}_{50}=7.1 \pm 0.05 \mu \mathrm{M}\right)$, 9, a 4-hydroxy analog $\left(\mathrm{IC}_{50}=5.3 \pm 0.1 \mu \mathrm{M}\right)$ and 13, a 2-hydroxy-4-methoxy analog $\left(\mathrm{IC}_{50}=12.3 \pm 0.3 \mu \mathrm{M}\right)$ also showed outstanding potential. The dihydroxy analogs are superior when mono-hydroxy analogs. The reason for greater potential of dihydroxy analogs is mainly due to greater number of hydroxyl group. This shows that number of hydroxyl group also paly critical role in this inhibition. In nitro substituted analogs like 10, a 2-nitro analog $\left(\mathrm{IC}_{50}\right.$ value $\left.24.38 \pm 0.3 \mu \mathrm{M}\right)$ is predominating over 11, a 3-nitro analog $\left(\mathrm{IC}_{50}\right.$ value $\left.35.30 \pm 0.5 \mu \mathrm{M}\right)$ and $\mathbf{1 2}$, a 4-nitro analog $\left(\mathrm{IC}_{50}\right.$ value $\left.28.21 \pm 0.4 \mu \mathrm{M}\right)$ which shows that position of substituent plays an important role. Similar pattern was also observed in other substituted analog like flouro analogs 18, a 2-nitro analog $\left(\mathrm{IC}_{50}\right.$ value $\left.4.6 \pm 0.2 \mu \mathrm{M}\right)$ with 19 , a 3 -nitro analog $\left(\mathrm{IC}_{50}\right.$ value $\left.9.9 \pm 0.4 \mu \mathrm{M}\right)$ and 20 , a 4-nitro analog $\left(\mathrm{IC}_{50}\right.$ value $6.2 \pm 0.3 \mu \mathrm{M})$ and chloro substituted analogs like compound 21, a 4-chloro analog $(12.0 \pm 0.30 \mu \mathrm{M})$ is more potent than 22, a 4-chloro analog $\left(\mathrm{IC}_{50}\right.$ value $19.28 \pm 0.3 \mu \mathrm{M}$ ) and 15, a 3-chloro analog $\left(\mathrm{IC}_{50}\right.$ value $19.8 \pm 0.3 \mu \mathrm{M})$. It was concluded form this study that position, nature and number of substituents on phenyl ring plays a critical in this inhibition.

\section{Molecular docking study}

The concentration inhibition $\mathrm{IC}_{50}$ values of thiadiazole synthesized derivatives as $\beta$-glucoronidase inhibitors are presented in (Table 1). As shown in Table 1, the inhibitory potency of the tilted compounds depends mainly on the type, number and positions of the functional group in the substitute group $\mathrm{R}$ of the synthesized derivatives. According to inhibitory $\mathrm{IC}_{50}$ values (Table 1), the synthesized derivatives may be subdivided into highly active group with low $\mathrm{IC}_{50}$ values (e.g., 6, 7, 3), moderate active group (e.g., 4, 9, 20) and low active group (e.g., 1, 2). For a better understanding of the observed results and to rationalize the highest activity of $\mathbf{6}$ compared to $\mathbf{4}$, and the low activity of $\mathbf{1}$ with regards to $\mathbf{4}$, molecular docking study has been carried out to shed light on the established binding modes of the three chosen synthesized compounds $\mathbf{6}, \mathbf{4}$ and $\mathbf{1}$ to the closest residues in the active site of $\beta$-glucoronidase enzyme. Table 2 summarized the calculated binding energies of the stable complexes ligand- $\beta$-glucoronidase, number of established intermolecular hydrogen bonding between the synthesized compounds (1, $\mathbf{4}$ and $\mathbf{6})$ and active site residues of $\beta$-glucoronidase. 
Table 1 Synthesis of indole based thiadiazole analogs and their $\beta$-glucuronidase potential

\begin{tabular}{|c|c|c|}
\hline S. No & $\mathbf{R}$ & $\mathrm{IC}_{50} \pm \mathrm{SEM}^{\mathrm{a}}$ \\
\hline 1 & & $12.60 \pm 0.1$ \\
\hline 2 & & $11.1 \pm 0.1$ \\
\hline 3 & & $3.1 \pm 0.01$ \\
\hline 4 & & $7.1 \pm 0.05$ \\
\hline 5 & & $1.82 \pm 0.01$ \\
\hline 6 & & $0.5 \pm 0.08$ \\
\hline 7 & & $1.1 \pm 0.08$ \\
\hline 8 & & $2.30 \pm 0.1$ \\
\hline 9 & & $5.3 \pm 0.1$ \\
\hline 10 & & $24.38 \pm 0.3$ \\
\hline 11 & & $35.30 \pm 0.5$ \\
\hline 12 & & $28.21 \pm 0.4$ \\
\hline 13 & & $12.3 \pm 0.3$ \\
\hline
\end{tabular}


Table 1 (continued)

\begin{tabular}{|c|c|c|}
\hline 14 & 8 & $38.9 \pm 0.8$ \\
\hline 15 & & $19.8 \pm 0.3$ \\
\hline 16 & & $19.7 \pm 0.5$ \\
\hline 17 & & $15.8 \pm 0.4$ \\
\hline 18 & & $4.6 \pm 0.2$ \\
\hline 19 & & $9.9 \pm 0.4$ \\
\hline 20 & & $6.2 \pm 0.3$ \\
\hline 21 & & $12.0 \pm 0.30$ \\
\hline 22 & $-\mathrm{Cl}$ & $19.28 \pm 0.3$ \\
\hline Standard & D-Saccharic acid 1,4 lactone ${ }^{b}$ & $48.1 \pm 1.2$ \\
\hline
\end{tabular}

a standard error mean

b standard drug<smiles>CCOC(=O)c1ccc2[nH]c(C(N)=O)cc2c1</smiles><smiles>NNC(=S)c1ccc2[nH]ccc2c1</smiles>

Substituted aldehydes $\mathrm{POCl} 3$, pyridine<smiles>[R]c1nnc(-c2ccc3[nH]ccc3c2)s1</smiles>

Scheme 1 Synthesis of indole based thiadiazole derivatives 1-22 
As can be seen from the docking results shown in Table 2 and Fig. 1, the highest activity of synthesized compound $\mathbf{6}$ compared to low active compounds $\mathbf{4}$ and $\mathbf{1}$ is mainly return to (ii) the stability of the formed complex between the docked compounds and $\beta$-glucoronidase, and the (ii) number of hydrogen bonding established between the docked ligands and the active site residues of the $\beta$-glucoronidase. However, it can be concluded that the number of closest residues to the docked ligands where all of them are surrounded by almost the same number of residues into the active site (Table 2 and Fig. 1) has no effect on the observed activities. Indeed, the formed complex between $\mathbf{6}$ and $\beta$-glucoronidase has the lowest binding energy of $-8.6 \mathrm{kcal} / \mathrm{mol}$ compared to 4 and 1 with binding energies of the stables complexes between the two compound and $\beta$-glucoronidase are of -8.3 and $-7.7 \mathrm{kcal} / \mathrm{mol}$, respectively (Table 2 ). In addition, four hydrogen bonding are established between residues of $\beta$-glucoronidase and compound $\mathbf{6}$ into the active site (Fig. 1c). The strongest hydrogen bond is formed between GLU45 amino acid and the hydrogen atom of hydroxyl group of catechol moiety of a $\mathbf{6}$ with distance of $1.66 \AA$. The second hydrogen bond is relatively weaker than the first one and is established between hydrogen atom of hydroxyl of catechol oxygen in ortho position of catechol group and GLU287 with a distance of $2.2 \AA$. The two other hydrogen bonds are relatively weak than the previous ones. The first one is established between ASN80 amino acid and the oxygen atom of the hydroxyl group of catechol moiety of $\mathbf{6}$ with a distance of $2.82 \AA$. The second one is established between HIS327 and hydrogen atom of hydroxyl group of catechol moiety of 6 with a distance of $2.96 \AA$. In a similar way, the higher activity of $\mathbf{4}$ compared to $\mathbf{1}$ may be explained by the above effects (i) and (ii) (Table 1 and Fig. 1). For instance, the complex formed between $\mathbf{4}$ and $\beta$-glucoronidase has a binding energy of $-8.3 \mathrm{kcal} / \mathrm{mol}$ and two hydrogen

Table 2 Concentration inhibition $I C_{50}$, docking binding energies and number of closest residues to the docked ligand in the active site of synthesized derivatives $(1,4$ and 6 ) within the active binding site of $\beta$-glucoronidase

\begin{tabular}{llllr}
\hline $\begin{array}{l}\text { No. } \\
\text { of compound }\end{array}$ & $\begin{array}{l}\text { Free } \\
\text { binding } \\
\text { energy } \\
\text { (kcal/mol) }\end{array}$ & $\begin{array}{l}\text { H-bonds } \\
\text { (HBs) }\end{array}$ & $\begin{array}{l}\text { Number } \\
\text { of closest } \\
\text { residues } \\
\text { to the docked } \\
\text { ligand } \\
\text { in the active } \\
\text { site }\end{array}$ & IC $_{\mathbf{5 0}} \pm$ SEM \\
\hline 1 & & & & \\
\hline 4 & -7.71 & 1 & 7 & $12.60 \pm 0.1$ \\
6 & -8.29 & 2 & 8 & $7.1 \pm 0.05$ \\
\hline
\end{tabular}

bonding of distance $1.95 \AA$, which are formed between amino acids ASP105 and TYR243 and hydrogen of NH and hydrogen atom of hydroxyl group of phenol group of $\mathbf{4}$, respectively. While, for the synthesized compound 1, the formed complex has energy binding of $-7.7 \mathrm{kcal} /$ $\mathrm{mol}$, and only one hydrogen bond that is formed between HIS241 amino acids and NH group of compound $\mathbf{1}$.

\section{Materials and methods}

NMR experiments were performed on Avance Bruker AM $300 \mathrm{MHz}$ machine. Electron impact mass spectra (EI MS) were recorded on a Finnigan MAT-311A (Germany) mass spectrometer. Thin layer chromatography (TLC) was performed on pre-coated silica gel aluminum plates (Kieselgel 60, 254, E. Merck, Germany). Chromatograms were visualized by UV at 254 and $365 \mathrm{~nm}$.

\section{Molecular docking details}

The interaction binding modes between the active site residues of $\beta$-glucoronidase and docked synthesized indole derivatives have been carried out using Autodock package [37-39]. X-ray coordinates of $\beta$-glucoronidase and the originated docked ligand $\mathrm{N}$-alkyl cyclophellitol aziridine were downloaded from the RCSB data bank web site (PDB code 5G0Q) [40-45]. Water molecules were removed; polar hydrogen atoms and Kollman charge were added to the extracted receptor structure by using the automated tool in AutoDock Tools 4.2. The active site is identified based on co-crystallized receptor-ligand complex structure of $\beta$-glucoronidase. The re-docking of the original ligand $N$-alkyl cyclophellitol aziridine into the active site is well reproduced with a RMSD value less than $2 \AA$. The molecular structures geometries of indole synthesized derivatives were minimized at Merck molecular force field 94 (MMFF94) level 44. The optimized structures were saved as pdb files. Nonpolar hydrogens were merged and rotatable bonds were defined for each docked ligand. Docking studies were performed by Lamarckian genetic algorithm, with 500 as total number of run for binding site for originated ligand and 100 run for the synthesized derivatives. In each respective run, a population of 150 individuals with 27,000 generations and 250,000 energy evaluations were employed. Operator weights for crossover, mutation, and elitism were set to $0.8,0.02$, and 1 , respectively. The binding site was defined using a grid of $40 \times 40 \times 40$ points each with a grid spacing of $0.375 \AA$. The docking calculation have been carried out using an Intel (R) Core (TM) i5-3770 CPU @ 3.40 GHz workstation (Additional file 1). 


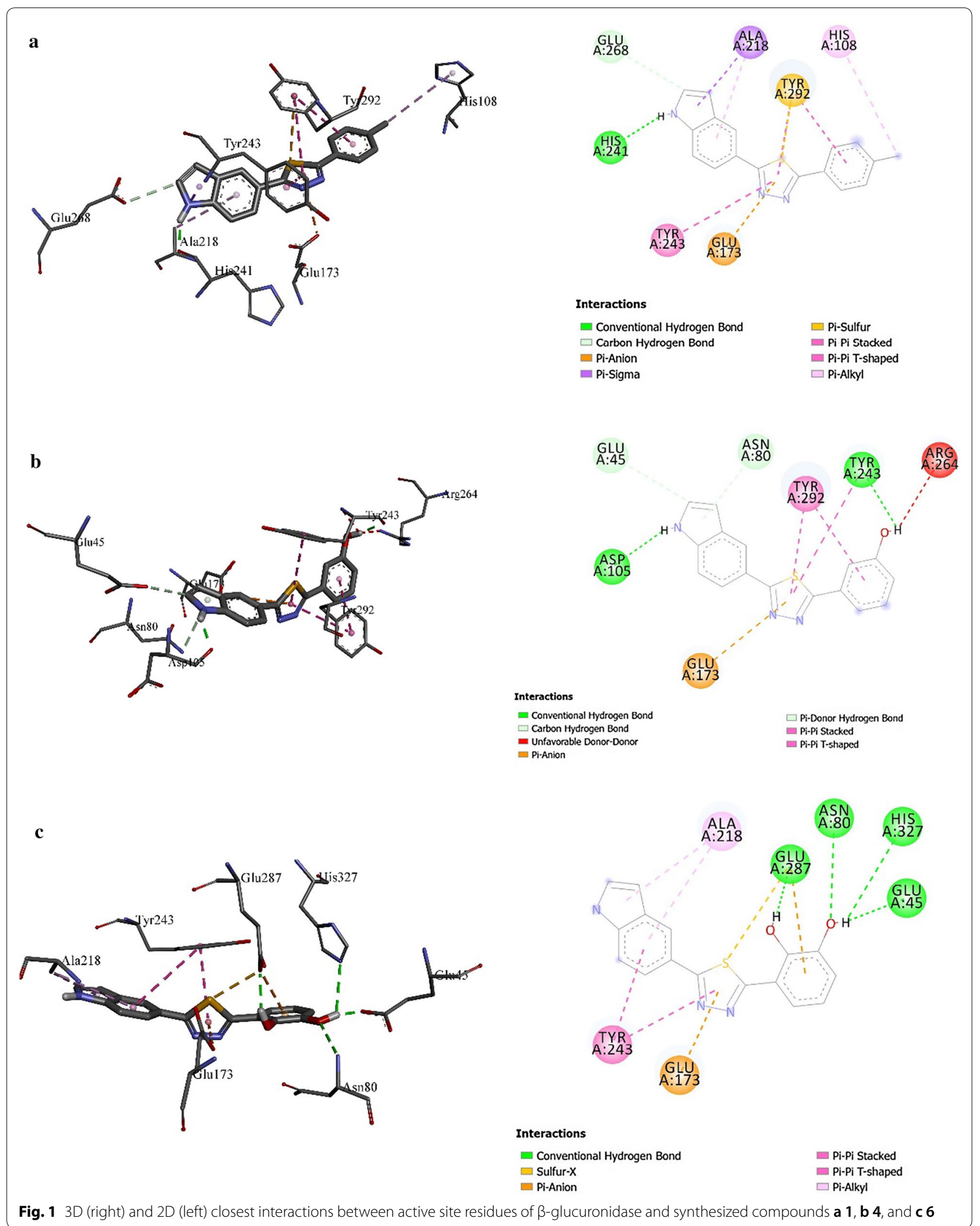




\section{General procedure for the synthesis of compounds (1-22)}

Thiadiazole derivatives 1-22 were synthesized by refluxing ethyl $1 H$-indole-5-carboxylate (a) with hydrazine hydrate in ethanol for $2 \mathrm{~h}$ to afford $1 \mathrm{H}$-indole-5-carbohydrazide (b) refluxed with Lawesson's reagent in toluene yielded corresponding thio-analogue (c). Thiohydrazide (c) was then treated with various aryl aldehydes to form cyclized adducts $\mathbf{1 - 2 2}$.

\section{Compound 1: 2-(1H-indol-5-yl)-5-(p-tolyl)-1,3,4-thiadiazole} Yield 90\%, ${ }^{1} \mathrm{H}-\mathrm{NMR}\left(500 \mathrm{MHz}, \mathrm{DMSO}-d_{6}\right): \delta 11.75(\mathrm{~s}$, $1 \mathrm{H}), 8.18$ (s, 1H), 7.68 (d, $1 \mathrm{H}, J=8.0 \mathrm{~Hz}), 7.60(\mathrm{~d}, 2 \mathrm{H}$, $J=6.5 \mathrm{~Hz}), 7.45(\mathrm{t}, 2 \mathrm{H}, J=8.0 \mathrm{~Hz}), 7.26(\mathrm{~d}, 2 \mathrm{H}, J=7.0 \mathrm{~Hz})$, $6.53(\mathrm{~s}, 1 \mathrm{H}), 2.38(\mathrm{~s}, 3 \mathrm{H}) ;{ }^{13} \mathrm{C}-\mathrm{NMR}(125 \mathrm{MHz}, \mathrm{DMSO}-$ $\left.d_{6}\right): \delta 173.8,173.4,133.3,131.4,130.3,130.1,129.5,129.4$, 128.4, 127.4, 127, 124.1, 119.4, 116.2, 111.2, 102.2, 21.1, EI-MS: $\mathrm{m} / \mathrm{z}$ calcd for $\mathrm{C}_{17} \mathrm{H}_{13} \mathrm{~N}_{3} \mathrm{~S}[\mathrm{M}]^{+}$291.0830, Found 291.0818 .

\section{Compound 2: 2-(1H-indol-5-yl)-5-(o-tolyl)-1,3,4-thiadiazole} Yield 87\%, ${ }^{1} \mathrm{H}-\mathrm{NMR}\left(500 \mathrm{MHz}, \mathrm{DMSO}-d_{6}\right): \delta 11.70(s$, $1 H), 8.23(\mathrm{~s}, 1 \mathrm{H}), 7.80(\mathrm{~d}, 1 \mathrm{H}, J=6.0 \mathrm{~Hz}), 7.68(\mathrm{~d}, 1 \mathrm{H}$, $J=8.0 \mathrm{~Hz}), 7.48-7.45(\mathrm{~m}, 2 \mathrm{H}), 7.29-7.26(\mathrm{~m}, 3 \mathrm{H}), 6.55$ (s, 1H), $2.43(\mathrm{~s}, 3 \mathrm{H}) ;{ }^{13} \mathrm{C}-\mathrm{NMR}\left(125 \mathrm{MHz}, \mathrm{DMSO}-d_{6}\right)$ : $\delta$ 173.8, 173.1, 137, 136.7, 135.3, 129.8, 129.4, 128.4, 128.3, 127.7, 126.3, 124.7, 119, 116.2, 111.2, 102.2, 18.5, EI-MS: $\mathrm{m} / \mathrm{z}$ calcd for $\mathrm{C}_{17} \mathrm{H}_{13} \mathrm{~N}_{3} \mathrm{~S}[\mathrm{M}]^{+}$291.0830, Found 291.0813 .

Compound 3: 2-(5-(1H-indol-5-yl)-1,3,4-thiadiazol-2-yl)phenol Yield 83\%, ${ }^{1} \mathrm{H}-\mathrm{NMR}\left(500 \mathrm{MHz}, \mathrm{DMSO}-d_{6}\right): \delta 11.90$ $(\mathrm{s}, 1 \mathrm{H}, \mathrm{NH}), 8.52(\mathrm{~s}, 1 \mathrm{H}, \mathrm{OH}), 8.20(\mathrm{~s}, 1 \mathrm{H}), 7.68(\mathrm{~d}$, $1 \mathrm{H}, J=8.0 \mathrm{~Hz}), 7.59(\mathrm{~d}, 1 \mathrm{H}, J=8.0 \mathrm{~Hz}), 7.52(\mathrm{~d}, 2 \mathrm{H}$, $J=7.5 \mathrm{~Hz}), 6.50(\mathrm{~d}, 2 \mathrm{H}, J=8.0 \mathrm{~Hz}), 6.57(\mathrm{~s}, 1 \mathrm{H}) ;{ }^{13} \mathrm{C}-\mathrm{NMR}$ $\left(125 \mathrm{MHz}, \mathrm{DMSO}-d_{6}\right): \delta 173.8,173,155.5,134.5,130.3$, 130, 129.3, 128.5, 124.0, 123.4, 121.5, 118.7, 117.5, 116.2, 111.3, 102.1, EI-MS: $\mathrm{m} / \mathrm{z}$ calcd for $\mathrm{C}_{16} \mathrm{H}_{11} \mathrm{~N}_{3} \mathrm{OS}[\mathrm{M}]^{+}$ 293.0623, Found 293.0609.

\section{Compound 4: 3-(5-(indol-5-yl)-1,3,4-thiadiazol-2-yl)phenol}

Yield 81\%, ${ }^{1} \mathrm{H}-\mathrm{NMR}\left(500 \mathrm{MHz}, \mathrm{DMSO}-d_{6}\right): \delta 9.60$ $(\mathrm{s}, 1 \mathrm{H}, \mathrm{NH}), 8.34(\mathrm{~s}, 1 \mathrm{H}, \mathrm{OH}), 8.18(\mathrm{~s}, 1 \mathrm{H}), 7.67(\mathrm{~d}$, $1 \mathrm{H}, J=8.0 \mathrm{~Hz}), 7.48(\mathrm{t}, 2 \mathrm{H}, J=7.5 \mathrm{~Hz}), 7.32(\mathrm{~d}, 1 \mathrm{H}$, $J=7.5 \mathrm{~Hz}), 7.24$ (d, $1 \mathrm{H}, J=7.5 \mathrm{~Hz}), 7.10$ (d, $1 \mathrm{H}$, $J=7.8), 6.83(\mathrm{~d}, 1 \mathrm{H}, J=7.5 \mathrm{~Hz}), 6.59(\mathrm{~s}, 1 \mathrm{H}) ;{ }^{13} \mathrm{C}-\mathrm{NMR}$ $\left(125 \mathrm{MHz}, \mathrm{DMSO}-d_{6}\right): \delta 174.0,174.0,157.3,151.4$, $134.7,130.4,129.7,128.0,126.6,123.3,121.6,115.7$, 113.7, 112.6, 46.6, 30.4, EI-MS: $\mathrm{m} / \mathrm{z}$ calcd for $\mathrm{C}_{16} \mathrm{H}_{12} \mathrm{~N}_{3} \mathrm{~S}$ $[\mathrm{M}]^{+}$293.0623, Found 293.0608.
Compound 5: 2-(5-(1H-indol-5-yl)-1,3,4-thiadiazol-2-yl) benzene-1,4-diol

Yield 80\%, ${ }^{1} \mathrm{H}-\mathrm{NMR}\left(500 \mathrm{MHz}, \mathrm{DMSO}-d_{6}\right): \delta 11.92(\mathrm{~s}$, $1 \mathrm{H}, \mathrm{NH}), 10.62$ (s, 1H, OH), 8.42 (s, 1H, OH), 8.31 (s, $1 \mathrm{H}), 7.70(\mathrm{~d}, 1 \mathrm{H}, J=8.0 \mathrm{~Hz}), 7.49(\mathrm{~d}, 2 \mathrm{H}, J=8.0 \mathrm{~Hz}), 6.75$ $(\mathrm{s}, 1 \mathrm{H}), 6.65(\mathrm{~s}, 1 \mathrm{H}), 6.55(\mathrm{~d}, 1 \mathrm{H}, J=8.0 \mathrm{~Hz}), 6.73(\mathrm{~d}, 1 \mathrm{H}$, $J=8.0 \mathrm{~Hz}), 6.56(\mathrm{~s}, 1 \mathrm{H}) ;{ }^{13} \mathrm{C}-\mathrm{NMR}(125 \mathrm{MHz}, \mathrm{DMSO}-$ $\left.d_{6}\right): \delta 174.0,174.0,150.0,147.5,135.3,129.5,128.5,125.0$, $124.1,118.8,117.6,117.1,116.2,114.1,111.4,102.2$, EI-MS: $\mathrm{m} / \mathrm{z}$ calcd for $\mathrm{C}_{16} \mathrm{H}_{11} \mathrm{~N}_{3} \mathrm{O}_{2} \mathrm{~S}[\mathrm{M}]^{+}$309.0572, Found 309.0554.

\section{Compound 6: 3-(5-(1H-indol-5-yl)-1,3,4-thiadiazol-2-yl)} benzene-1,2-diol

Yield 88\%, ${ }^{1} \mathrm{H}-\mathrm{NMR}\left(500 \mathrm{MHz}, \mathrm{DMSO}-d_{6}\right): \delta 12.08$ (s, 1H, NH), 9.14 (s, 1H, OH), $8.55(\mathrm{~s}, 1 \mathrm{H}, \mathrm{OH}), 8.20$ (s, $1 \mathrm{H}), 7.70(\mathrm{~d}, 1 \mathrm{H}, J=8.0 \mathrm{~Hz}), 7.55(\mathrm{~d}, 1 \mathrm{H}, J=8.0 \mathrm{~Hz})$, $6.90(\mathrm{~d}, 1 \mathrm{H}, J=7.8 \mathrm{~Hz}), 6.85(\mathrm{~d}, 1 \mathrm{H}, J=7.8), 6.76(\mathrm{t}, 1 \mathrm{H}$, $J=7.0 \mathrm{~Hz}), 6.60$ (d, $1 \mathrm{H}, J=2.0 \mathrm{~Hz}) ;{ }^{13} \mathrm{C}-\mathrm{NMR}(125 \mathrm{MHz}$, DMSO- $\left.d_{6}\right): \delta$ 174.0. 174.0, 145.4, 143.7, 135.3, 129.5, $128.5,125.0,124.1,123.0,121.3,118.8,117.1,116.2$, 111.4, 102.2, EI-MS: $\mathrm{m} / \mathrm{z}$ calcd for $\mathrm{C}_{16} \mathrm{H}_{11} \mathrm{~N}_{3} \mathrm{O}_{2} \mathrm{~S}[\mathrm{M}]^{+}$ 309.0572, Found 309.0550.

\section{Compound 7: 4-(5-(1H-indol-5-yl)-1,3,4-thiadiazol-2-yl)} benzene-1,2-diol

Yield 77\%, ${ }^{1} \mathrm{H}-\mathrm{NMR}\left(500 \mathrm{MHz}, \mathrm{DMSO}-d_{6}\right): \delta 9.32(\mathrm{~s}, 1 \mathrm{H}$, $\mathrm{NH}), 9.21$ (s, 1H, OH), $8.32(\mathrm{~s}, 1 \mathrm{H}, \mathrm{OH}), 8.6(\mathrm{~s}, 1 \mathrm{H}), 7.71$ $(\mathrm{d}, 1 \mathrm{H}, J=8.0 \mathrm{~Hz}), 7.42(\mathrm{~d}, 2 \mathrm{H}, J=8.0 \mathrm{~Hz}), 7.23(\mathrm{~s}, 1 \mathrm{H})$, $6.91(\mathrm{~d}, 1 \mathrm{H}, J=7.0 \mathrm{~Hz}), 6.74(\mathrm{~d}, 1 \mathrm{H}, J=8.0 \mathrm{~Hz}), 6.56(\mathrm{~d}$, $1 \mathrm{H}, J=2.0 \mathrm{~Hz}) ;{ }^{13} \mathrm{C}-\mathrm{NMR}\left(125 \mathrm{MHz}, \mathrm{DMSO}-d_{6}\right): \delta 174.0$, $174.0,147.1,145.7,135.4,129.5,128.5,127.3,124.1$, 121.3, 118.8, 116.2, 116.0, 14.1, 111.4, 102.2, EI-MS: m/z calcd for $\mathrm{C}_{16} \mathrm{H}_{11} \mathrm{~N}_{3} \mathrm{O}_{2} \mathrm{~S}[\mathrm{M}]^{+}$309.0572, Found 309.0559.

\section{Compound 8: 4-(5-(1H-indol-5-yl)-1,3,4-thiadiazol-2-yl)} benzene-1,3-diol

Yield 73\%, ${ }^{1} \mathrm{H}-\mathrm{NMR}\left(500 \mathrm{MHz}, \mathrm{DMSO}-d_{6}\right): \delta 11.80(\mathrm{~s}, 1 \mathrm{H}$, $\mathrm{NH}), 9.92(\mathrm{~s}, 1 \mathrm{H}, \mathrm{OH}), 8.53(\mathrm{~s}, 1 \mathrm{H}, \mathrm{OH}), 8.18$ (s, 1H), 7.71 $(\mathrm{d}, 1 \mathrm{H}, J=8.0 \mathrm{~Hz}), 7.47(\mathrm{~d}, 2 \mathrm{H}, J=8.0 \mathrm{~Hz}), 7.44(\mathrm{t}, 1 \mathrm{H}$, $J=6.0 \mathrm{~Hz}), 7.25(\mathrm{~d}, 1 \mathrm{H}, J=8.0), 6.34(\mathrm{~d}, 1 \mathrm{H}, J=8.0 \mathrm{~Hz})$, 6.31(d, $1 \mathrm{H}, J=6.0 \mathrm{~Hz}) ;{ }^{13} \mathrm{C}-\mathrm{NMR}\left(125 \mathrm{MHz}, \mathrm{DMSO}-d_{6}\right)$ : $\delta$ 174.0, 174.0, 159.7, 156.4, 135.3, 130.1, 129.5, 128.5, $124.1,118.8,116.2,116.1,111.4,108.9,105.4,102.2$, EI-MS: $\mathrm{m} / \mathrm{z}$ calcd for $\mathrm{C}_{16} \mathrm{H}_{11} \mathrm{~N}_{3} \mathrm{O}_{2} \mathrm{~S}[\mathrm{M}]^{+}$309.0572, Found 309.0558.

Compound 9: 4-(5-(1H-indol-5-yl)-1,3,4-thiadiazol-2-yl)phenol Yield 79\%, ${ }^{1} \mathrm{H}-\mathrm{NMR}\left(500 \mathrm{MHz}, \mathrm{DMSO}-d_{6}\right): \delta 11.59$ (s, 1H, NH), 8.39 (s, 1H, OH), $8.16(\mathrm{~S}, 1 \mathrm{H}), 7.65$ (d, 1H, $J=8.0 \mathrm{~Hz}), 7.54(\mathrm{~d}, 2 \mathrm{H}, J=8.0 \mathrm{~Hz}), 7.45-7.42(\mathrm{~m}, 2 \mathrm{H})$, $6.80(\mathrm{~d}, 2 \mathrm{H}, J=8.0 \mathrm{~Hz}), 6.55(\mathrm{~s}, 1 \mathrm{H}) ;{ }^{13} \mathrm{C}-\mathrm{NMR}(125 \mathrm{MHz}$, 
DMSO- $\left.d_{6}\right): \delta 174.0,174.0,158.3,135.3,129.5,128.7$, $128.7,128.5,126.0,124.1,118.8,116.2,116.2,116.2$, 111.4, 102.2, EI-MS: $\mathrm{m} / \mathrm{z}$ calcd for $\mathrm{C}_{16} \mathrm{H}_{11} \mathrm{~N}_{3} \mathrm{OS}[\mathrm{M}]^{+}$ 293.0623, Found 293.0627.

\section{Compound 10: 2-(1H-indol-5-yl)-5-(2-nitrophenyl)-1,3,4- thiadiazole}

Yield 81\%, ${ }^{1} \mathrm{H}-\mathrm{NMR}$ (500 MHz, DMSO-d6): $\delta 12.10$ (s, $1 \mathrm{H}, \mathrm{NH}), 8.23(\mathrm{~s}, 1 \mathrm{H}), 8.14(\mathrm{~d}, 1 \mathrm{H}, J=7.0 \mathrm{~Hz}), 8.05(\mathrm{~d}, 1 \mathrm{H}$, $J=8.0 \mathrm{~Hz}), 7.82(\mathrm{t}, 1 \mathrm{H}, J=7.0 \mathrm{~Hz}), 7.70(\mathrm{~d}, 1 \mathrm{H}, J=8.0)$, $7.65(\mathrm{t}, 1 \mathrm{H}, J=7.0 \mathrm{~Hz}), 7.51-7.48(\mathrm{~m}, 2 \mathrm{H}) ; 6.72(\mathrm{~s}, 1 \mathrm{H})$; ${ }^{13} \mathrm{C}$ NMR (125 MHz, DMSO-d6): $\delta$ 174.0, 174.0, 146.7, $135.3,135.1,131.4,129.5,129.4,128.5,128.2,124.2$, 124.1, 118.8, 116.2, 111.4, 102.2, EI-MS: $\mathrm{m} / \mathrm{z}$ calcd for $\mathrm{C}_{16} \mathrm{H}_{10} \mathrm{~N}_{4} \mathrm{O}_{2} \mathrm{~S}[\mathrm{M}]^{+}$322.0524, Found 322.0510.

\section{Compound 11: 2-(1H-indol-5-yl)-5-(3-nitrophenyl)-1,3,4- thiadiazole}

Yield 89\%, ${ }^{1} \mathrm{H}-\mathrm{NMR}$ (500 MHz, DMSO-d6): $\delta 12.08$ (s, $1 \mathrm{H}), 8.59$ (d, 2H, J=8.5 Hz), 8.25-8.22 (m, 2H), 8.14 $(\mathrm{d}, 1 \mathrm{H}, J=7.0 \mathrm{~Hz}), 7.74(\mathrm{t}, 1 \mathrm{H}, J=8.0 \mathrm{~Hz}), 7.70(\mathrm{~d}, 1 \mathrm{H}$, $J=8.0 \mathrm{~Hz}), 7.50-7.46(\mathrm{~m}, 2 \mathrm{H}), 6.63(\mathrm{~s}, 1 \mathrm{H}) ;{ }^{13} \mathrm{C} \mathrm{NMR}$ (125 MHz, DMSO-d6): $\delta$ 174.0, 174.0, 148.2, 137.0, 135.3, $134.2,130.0,129.5,128.5,124.1,123.7,122.6,118.8$, 116.2, 111.4, 102.2, EI-MS: $\mathrm{m} / \mathrm{z}$ calcd for $\mathrm{C}_{16} \mathrm{H}_{10} \mathrm{~N}_{4} \mathrm{O}_{2} \mathrm{~S}$ $[\mathrm{M}]^{+}$322.0524, Found 322.0507.

\section{Compound 12: 2-(1H-indol-5-yl)-5-(4-nitrophenyl)-1,3,4- thiadiazole}

Yield 91\%, ${ }^{1} \mathrm{H}-\mathrm{NMR}$ (500 MHz, DMSO-d6): $\delta 12.03$ (s, 1H, NH), 8.30 (d, 2H, J=8.0 Hz), $8.22(\mathrm{~s}, 1 \mathrm{H}), 8.15$ (d, $1 \mathrm{H}, J=8.0 \mathrm{~Hz}), 8.01(\mathrm{~d}, 2 \mathrm{H}, J=8.0 \mathrm{~Hz}), 7.70(\mathrm{~d}, 1 \mathrm{H}$, $J=8.0 \mathrm{~Hz}), 7.49-7.46(\mathrm{~m}, 2 \mathrm{H}) 6.62(\mathrm{~s}, 1 \mathrm{H}) ;{ }^{13} \mathrm{C} \mathrm{NMR}$ (125 MHz, DMSO-d6): $\delta$ 174.7, 174, 148.1, 139.2, 135.2, $129.5,128.6,128.7,128.2,124.8,124.5,124,119.3,116$, 111.2, 102, EI-MS: $\mathrm{m} / \mathrm{z}$ calcd for $\mathrm{C}_{16} \mathrm{H}_{10} \mathrm{~N}_{4} \mathrm{O}_{2} \mathrm{~S}[\mathrm{M}]^{+}$ 322.0524, Found 322.0509.

\section{Compound 13: 2-(5-(1H-indol-5-yl)-1,3,4-thiadiazol-2-yl)-4- methoxyphenol}

Yield 90\%, ${ }^{1} \mathrm{H}-\mathrm{NMR}$ (500 MHz, DMSO-d6): $\delta 12.08$ (s, $1 \mathrm{H}, \mathrm{NH}), 11.41$ (s, $1 \mathrm{H}, \mathrm{OH}), 8.23(\mathrm{~s}, 1 \mathrm{H}), 7.70(\mathrm{~d}, 1 \mathrm{H}$, $J=8.0 \mathrm{~Hz}), 7.49-7.46(\mathrm{~m}, 2 \mathrm{H}), 7.09(\mathrm{~d}, 1 \mathrm{H}, J=8.0 \mathrm{~Hz})$, $6.90(\mathrm{~d}, 2 \mathrm{H}, J=2.0 \mathrm{~Hz}), 6.84(\mathrm{~d}, 1 \mathrm{H}, J=7.5 \mathrm{~Hz}), 3.78(\mathrm{~s}$, $3 \mathrm{H}) ;{ }^{13} \mathrm{C}$ NMR (125 MHz, DMSO-d6): $\delta$ 174.7, 174.0, $153.5,147.1,135.8,129.9,128.6,124.9,124.4,119.3$, 117.2, 116.2, 115.4, 112.5, 111.3, 102.2, 55.4, EI-MS: $\mathrm{m} / \mathrm{z}$ calcd for $\mathrm{C}_{17} \mathrm{H}_{13} \mathrm{~N}_{3} \mathrm{O}_{2} \mathrm{~S}[\mathrm{M}]^{+}$323.0728, Found 323.0711.

Compound 14: 2-(3-bromo-4-fluorophenyl)-5-(1H-indol-5-yl)1,3,4-thiadiazole

Yield 88\%, ${ }^{1} \mathrm{H}-\mathrm{NMR}$ (500 MHz, DMSO-d6): $\delta$ 11.94 (s, $1 \mathrm{H}), 8.20(\mathrm{~s}, 1 \mathrm{H}), 8.02(\mathrm{~d}, 1 \mathrm{H}, J=7.0 \mathrm{~Hz}), 7.75(\mathrm{~s}, 1 \mathrm{H}), 7.67$ (d, 2H, J=7.0 Hz), 7.48-7.45 (m, 3H), $6.65(\mathrm{~s}, 1 \mathrm{H}) ;{ }^{13} \mathrm{C}$ NMR (125 MHz, DMSO-d6): $\delta$ 174.4, 174.3, 165.2, 135.3, $134.4,131,129.4,128.5,128.8,124.7,119.3,118,116.3$, 111.3, 110.4, 102.2, EI-MS: $\mathrm{m} / \mathrm{z}$ calcd for $\mathrm{C}_{16} \mathrm{H}_{9} \mathrm{BrFN}_{3} \mathrm{~S}$ $[\mathrm{M}]^{+}$372.9685, Found 372.9661.

\section{Compound 15: 2-(3-chlorophenyl)-5-(1H-indol-5-yl)-1,3,4- thiadiazole}

Yield 90\%, ${ }^{1} \mathrm{H}$-NMR (500 MHz, DMSO-d6): $\delta 9.72(\mathrm{~s}, 1 \mathrm{H}$, $\mathrm{NH}), 8.20(\mathrm{~s}, 1 \mathrm{H}), 7.78(\mathrm{~d}, 1 \mathrm{H}, J=7.0 \mathrm{~Hz}), 7.48-7.45(\mathrm{~m}$, $2 \mathrm{H}), 7.35-7.31(\mathrm{~m}, 2 \mathrm{H}), 7.12(\mathrm{~d}, 1 \mathrm{H}, J=7.0 \mathrm{~Hz}), 6.82(\mathrm{~d}$, $1 \mathrm{H}, J=8.0 \mathrm{~Hz}), 6.62(\mathrm{~s}, 1 \mathrm{H}) ;{ }^{13} \mathrm{C}$ NMR $(125 \mathrm{MHz}, \mathrm{DMSO}-$ d6): $\delta 174.7,174.4,135.3,135.1,134.5,129.5,129.4,129.3$, $128.5,128.3,127.2,124.1,119.3,116.2,111.7,102.9$, EI-MS: $\mathrm{m} / \mathrm{z}$ calcd for $\mathrm{C}_{16} \mathrm{H}_{10} \mathrm{ClN}_{3} \mathrm{~S}[\mathrm{M}]^{+} 311.0284$, Found 311.0280 .

\section{Compound 16: 2-(1H-indol-5-yl)-5-(pyridin-3-yl)-1,3,4- thiadiazole}

Yield 81\%, ${ }^{1} \mathrm{H}-\mathrm{NMR}$ (500 MHz, DMSO-d6): $\delta 11.90(\mathrm{~s}$, $1 \mathrm{H}), 8.62(\mathrm{~s}, 1 \mathrm{H}), 8.58(\mathrm{~d}, 1 \mathrm{H}, J=5.0 \mathrm{~Hz}), 8.24(\mathrm{~s}, 1 \mathrm{H})$, $8.12(\mathrm{~d}, 1 \mathrm{H}, J=6.5 \mathrm{~Hz}), 7.70(\mathrm{~d}, 1 \mathrm{H}, J=8.0 \mathrm{~Hz}), 7.46-7.42$ (m, 3H), 6.58 (s, 1H); ${ }^{13} \mathrm{C}$ NMR (125 MHz, DMSO-d6): $\delta$ 174.4, 174, 149.3, 148.1, 135.2, 134.3, 133.2, 129.5, 128.4, 124.4, 124.3, 119.2, 116.2, 111.4, 102.6, EI-MS: m/z calcd for $\mathrm{C}_{15} \mathrm{H}_{10} \mathrm{~N}_{4} \mathrm{~S}[\mathrm{M}]^{+}$278.0626, Found 278.0612.

\section{Compound 17: 2-(1H-indol-5-yl)-5-(pyridin-4-yl)-1,3,4- thiadiazole}

Yield 80\%, ${ }^{1} \mathrm{H}-\mathrm{NMR}(500 \mathrm{MHz}, \mathrm{DMSO}-d 6): \delta 12.01$ $(\mathrm{s}, 1 \mathrm{H}), 8.62(\mathrm{~d}, 2 \mathrm{H}, J=6.5 \mathrm{~Hz}), 8.48(\mathrm{~s}, 1 \mathrm{H}), 7.70(\mathrm{~d}$, $1 \mathrm{H}, J=8.0 \mathrm{~Hz}), 7.68(\mathrm{~d}, 2 \mathrm{H}, J=8.0 \mathrm{~Hz}), 7.57(\mathrm{~d}, 1 \mathrm{H}$, $J=4.5 \mathrm{~Hz}), 7.50(\mathrm{~d}, 1 \mathrm{H}, J=8.0 \mathrm{~Hz}), 6.66(\mathrm{~s}, 1 \mathrm{H}) ;{ }^{13} \mathrm{C} \mathrm{NMR}$ (125 MHz, DMSO-d6): $\delta$ 174.5, 174, 150.1, 149.5, 143.5, 135.3, 129.4, 128.4, 124.1, 121.4, 121.2, 119.2, 116.2, 111.4, 102.7, EI-MS: $\mathrm{m} / \mathrm{z}$ calcd for $\mathrm{C}_{15} \mathrm{H}_{10} \mathrm{~N}_{4} \mathrm{~S}[\mathrm{M}]+278.0626$, Found 278.0614.

\section{Compound 18: 2-(2-fluorophenyl)-5-(1H-indol-5-yl)-1,3,4- thiadiazole}

Yield 89\%, ${ }^{1} \mathrm{H}-\mathrm{NMR}$ (500 MHz, DMSO-d6): $\delta 11.88$ (s, $1 \mathrm{H}, \mathrm{NH}), 8.22(\mathrm{~s}, 1 \mathrm{H}), 8.08(\mathrm{~s}, 1 \mathrm{H}), 7.70(\mathrm{~d}, 1 \mathrm{H}, J=8.0 \mathrm{~Hz})$, $7.50(\mathrm{~d}, 1 \mathrm{H}, J=8.0 \mathrm{~Hz}), 7.42(\mathrm{t}, 1 \mathrm{H}, J=6.5 \mathrm{~Hz}), 7.38(\mathrm{t}, 1 \mathrm{H}$, $J=6.5 \mathrm{~Hz}), 7.29(\mathrm{~d}, 2 \mathrm{H}, J=8.0 \mathrm{~Hz}), 6.68(\mathrm{~s}, 1 \mathrm{H}) ;{ }^{13} \mathrm{C} \mathrm{NMR}$ (125 MHz, DMSO-d6): $\delta 174.4,174.2,158.1,135.2,130.1$, $129.5,129.4,128.6,124.7,124.4,123.4,119.1,116.5$, 114.8, 111.4, 102.2, EI-MS: $\mathrm{m} / \mathrm{z}$ calcd for $\mathrm{C}_{16} \mathrm{H}_{10} \mathrm{FN}_{3} \mathrm{~S}$ $[\mathrm{M}]+295.0559$, Found 295.0574.

\section{Compound 19: 2-(3-fluorophenyl)-5-(1H-indol-5-yl)-1,3,4- thiadiazole}

Yield 83\%, ${ }^{1} \mathrm{H}-\mathrm{NMR}$ (500 MHz, DMSO-d6): $\delta 11.84$ $(\mathrm{s}, 1 \mathrm{H}, \mathrm{NH}), 8.44(\mathrm{~s}, 1 \mathrm{H}), 8.20(\mathrm{~s}, 1 \mathrm{H}), 7.68(\mathrm{~d}, 1 \mathrm{H}$, 
$J=8.0 \mathrm{~Hz}), 7.47-7.42(\mathrm{~m}, 4 \mathrm{H}), 7.26(\mathrm{t}, 1 \mathrm{H}, J=8.0 \mathrm{~Hz}), 6.66$ (s, $1 \mathrm{H}) ; \delta{ }^{13} \mathrm{C}$ NMR (125 MHz, DMSO-d6): $\delta$ 174.6, 174.2, $162.4,135.8,135.6,129.5,128.4,127.3,126.2,124.1$, 119.3, 116.2, 116.2, 115.3, 111.4, 102.2, EI-MS: m/z calcd for $\mathrm{C}_{16} \mathrm{H}_{10} \mathrm{FN}_{3} \mathrm{~S}[\mathrm{M}]+295.0579$, Found 295.057.

\section{Compound 20: 2-(4-fluorophenyl)-5-(1H-indol-5-yl)-1,3,4-} thiadiazole

Yield 88\%, ${ }^{1} \mathrm{H}-\mathrm{NMR}$ (500 MHz, DMSO-d6): $\delta 11.82$ (s, $1 \mathrm{H}, \mathrm{NH}), 8.15(\mathrm{~s}, 1 \mathrm{H}), 7.64(\mathrm{~d}, 2 \mathrm{H}, J=8.0 \mathrm{~Hz}), 7.49(\mathrm{t}, 1 \mathrm{H}$, $J=8.0 \mathrm{~Hz}), 7.47(\mathrm{t}, 2 \mathrm{H}, J=5.5 \mathrm{~Hz}), 7.31(\mathrm{t}, 2 \mathrm{H}, J=8.0 \mathrm{~Hz})$, $6.58(\mathrm{~s}, 1 \mathrm{H}) ;{ }^{13} \mathrm{C}$ NMR (125 MHz, DMSO-d6): $\delta$ 174.4, $174.2,162.7,135.2,129.4,129.4,129.2,129,128.5,124.1$, 119.6, 117.2, 116.7, 116.2, 111.4, 102.2, EI-MS: m/z calcd for $\mathrm{C}_{16} \mathrm{H}_{10} \mathrm{FN}_{3} \mathrm{~S}[\mathrm{M}]+295.0579$, Found 295.0574.

\section{Compound 21: 2-(2-chlorophenyl)-5-(1H-indol-5-yl)-1,3,4- thiadiazole}

Yield 83\%, ${ }^{1} \mathrm{H}-\mathrm{NMR}$ (500 MHz, DMSO-d6): $\delta 12.03(\mathrm{~s}$, $1 \mathrm{H}, \mathrm{NH}), 8.23(\mathrm{~s}, 1 \mathrm{H}), 8.05(\mathrm{~s}, 1 \mathrm{H}), 7.70(\mathrm{~d}, 1 \mathrm{H}, J=8.0 \mathrm{~Hz})$, $7.50(\mathrm{t}, 1 \mathrm{H}, J=8.0 \mathrm{~Hz}), 7.47(\mathrm{~d}, 1 \mathrm{H}, J=8.0 \mathrm{~Hz}), 7.42(\mathrm{t}, 1 \mathrm{H}$, $J=5.0 \mathrm{~Hz}), 7.39(\mathrm{~d}, 2 \mathrm{H}, J=6.5 \mathrm{~Hz}), 6.56(\mathrm{~s}, 1 \mathrm{H}) ;{ }^{13} \mathrm{C} \mathrm{NMR}$ (125 MHz, DMSO-d6): $\delta 174.7,174.3,137.1,135.3,132.0$, $130.3,129.5,129.5,129.2,128.5,127.1,124.2,119.1$, 116.2, 111.4, 102.2, EI-MS: $\mathrm{m} / \mathrm{z}$ calcd for $\mathrm{C}_{16} \mathrm{H}_{10} \mathrm{ClN}_{3} \mathrm{~S}$ $[\mathrm{M}]^{+}$311.0284, Found 311.0267.

\section{Compound 22: 2-(4-chlorophenyl)-5-(1H-indol-5-yl)-1,3,4- thiadiazole}

Yield 81\%, ${ }^{1} \mathrm{H}-\mathrm{NMR}$ (500 MHz, DMSO-d6): $\delta 11.80$ (s, 1H, NH), $8.20(\mathrm{~s}, 1 \mathrm{H}), 7.74(\mathrm{~d}, 2 \mathrm{H}, J=7.8 \mathrm{~Hz}), 7.68$ (d, $1 \mathrm{H}, J=8.0 \mathrm{~Hz}), 7.52(\mathrm{~d}, 2 \mathrm{H}, J=8.0 \mathrm{~Hz}), 7.48(\mathrm{~d}, 1 \mathrm{H}$, $J=8.0 \mathrm{~Hz}), 7.43(\mathrm{t}, 1 \mathrm{H}, J=6.0 \mathrm{~Hz}), 6.58(\mathrm{~s}, 1 \mathrm{H}) ;{ }^{13} \mathrm{C} \mathrm{NMR}$ (125 MHz, DMSO-d6): $\delta$ 174.6, 174.3, 135.3, 134.1, 131.4, $129.5,129.4,129.2,129.0,128.7,128.4,124.1,119.3$, 116.2, 111.4, 102.2, EI-MS: m/z calcd for $\mathrm{C}_{16} \mathrm{H}_{10} \mathrm{ClN}_{3} \mathrm{~S}$ $[\mathrm{M}]^{+}$311.0284, Found 311.0260.

\section{Conclusion}

In conclusion we have synthesized 22 derivatives of indole based thiadiazole hybrid analogs due to greater biological importance of indole and thiadiazole and evaluated for $\beta$-glucuronidase inhibition. All compounds showed outstanding $\beta$-glucuronidase activity ranging between $0.5 \pm 0.08$ to $38.9 \pm 0.8 \mu \mathrm{M}$ when compared with standard D-saccharic acid 1,4 lactone. Structure activity relationship has been established for all compounds which reveal that the number, nature and position of substituents on phenyl ring play of thiadiazole play a vital role in this inhibition. Molecular docking study have been performed which revealed that these compounds established stronger hydrogen bonding networks with active site residues.

\section{Additional file}

Additional file 1. Supporting data having proton NMR of all compounds.

\section{Authors' contributions}

NBA synthesized all compounds and wrote experimental part of manuscript. MT supervised the work and help in characterizing compounds. MG supported computational work and revised manuscript. FR helped in purifying compounds and manuscript writing. MI helped in bioassay work and writing SAR of all compounds. AM helped in calculating $I C_{50}$ and deeply analyzing bioactivity. EHA performed Molecular docking study and helped in writing manuscript. All authors read and approved the final manuscript.

\section{Author details}

${ }^{1}$ Department of Clinical Pharmacy, Institute for Research and Medical Consultations (IRMC), Imam Abdulrahman Bin Faisal University, P. O. Box 1982, Dammam 31441, Saudi Arabia. ${ }^{2}$ Department of Chemistry, Hazara University, Mansehra 21300, Khyber Pakhtunkhwa, Pakistan. ${ }^{3}$ Department of Computer Information Systems, College of Computer Science \& Information Technology, Imam Abdulrahman Bin Faisal University, P. O. Box 1982, Dammam 31441, Saudi Arabia. ${ }^{4}$ Department of Chemistry, College of Sciences and Humanities, Prince Sattam Bin Abdulaziz University, P.O. Box 83, Al-Kharj 11942, Saudi Arabia.

\section{Acknowledgements}

Authors thanks to Imam Abdulrahman Bin Faisal University for support and providing lab Facilities.

\section{Competing interests}

The authors declare that they have no competing interests.

Availability of data and materials

Data and materials are available.

Funding

There is no funding for this study.

\section{Publisher's Note}

Springer Nature remains neutral with regard to jurisdictional claims in published maps and institutional affiliations.

Received: 8 November 2018 Accepted: 16 January 2019 Published online: 04 February 2019

\section{References}

1. Jain S, Drendel WB, Chen ZW, Mathews FS, Sly WS, Grubb JH (1996) Structure of human beta-glucuronidase reveals candidate lysosomal targeting and active-site motifs. Nat Struct Biol 3:375-381

2. Wallace BD, Wang HW, Lane KT, Scott JE, Orans J, Koo JS, Venkatesh M, Jobin C, Yeh LA, Mani S, Redinbo MR (2010) Alleviating cancer drug toxicity by inhibiting a bacterial enzyme. Science 330:831-835

3. Michikawa M, Ichinose $H$, Momma M, Biely $P$, Jongkees S, Yoshida M, Kotake T, Tsumuraya Y, Withers SG, Fujimoto Z, Kaneko S (2012) Structural and biochemical characterization of glycoside hydrolase family 79 beta-glucuronidase from Acidobacterium capsulatum. J Biol Chem 287:14069-14077

4. Sakurama H, Kishino S, Uchibori Y, Yonejima Y, Ashida H, Kita K, Takahashi S, Ogawa J (2014) Beta-Glucuronidase from Lactobacillus brevis useful 
for baicalin hydrolysis belongs to glycoside hydrolase family 30 . Appl Microbiol Biotechnol 98:4021-4032

5. Song X, Jiang Z, Li L, Wu H (2014) Immobilization of â-glucuronidase in lysozyme-induced biosilica particles to improve its stability. Front Chem Sci Eng 8:353-436

6. Glaser JH, Sly WS (1973) b-Glucuronidase deficiency mucopolysaccharidosis: methods for enzymatic diagnosis. J Lab Clin Med 82:969-977

7. Holiman PCH, Hertog MGL, Katan MB (1996) Analysis and health effects of flavonoids. Food Chem 57:43-46

8. Ho KJ, Hsu SC, Chen JS, Ho LHC (1986) Human biliary glucuronidase: correlation of its activity with deconjugation of bilirubin in the bile. Eur J Clin Invest 16:361-367

9. Gonick HC, Kramer HJ, Schapiro AE (1973) Urinary ß-glucuronidase activity in renal disease. Arch Intern Med 132:63-69

10. Sly WS, Quinton BA, McAlister WH, Rimoin DL (1973) $\beta$-Glucuronidase deficiency: report of clinical, radiologic, and biochemical features of a new mucopolysaccharidosis. J Pediatr 82:249-257

11. Hall CW, Cantz M, Neufeld EF (1973) $\beta$-Glucuronidase deficiency mucopolysaccharidosis: studies in cultured fibroblasts. Arch Biochem Biophys 155:32-38

12. Cacchi S, Fabrizi G (2005) Synthesis and functionalization of indoles through palladiumcatalyzed reactions. Chem Rev 105:2873-2920

13. Imran S, Taha M, Ismail NH (2015) A review of bisindolylmethane as an important scaffold for drug discovery. Curr Med Chem 22:4412-4433

14. Haider N, Sotelo E (2002) 1,5-Dimethyl-6 h-pyridazino [4,5-b] carbazole, a 3 -aza bioisoster of the antitumor alkaloid olivacine. Chem Pharm Bull 50:1479-1483

15. Sakai S, Aimi N, Yamaguchi K, Hitotsuyanagi Y, Watanabe C, Yokose K, Koyama Y, Shudo K, Itai A (1984) Elucidation of the structure of olivoretin $\mathrm{a}$ and d(teleocidin B). Chem Pharm Bull 32:354-357

16. Xu YC, Schaus JM, Walker C, Krushinski J, Adham N, Zgombick JM, Liang SX, Kohlman DT, Audia JE (1999) N-Methyl-5-tert-butyltryptamine: a novel, highly potent 5-HT1D receptor agonist. J Med Chem 42:526-531

17. Moloney GP, Martin GR, Mathews N, Milne A, Hobbs H, Dodsworth S, Sang PY, Knight C, Williams M, Maxwell M (1999) Synthesis and serotonergic activity of substituted 2-N-benzylcarboxamido-5-(2-ethyl1-dioxoimidazolidinyl)-N,N-dimethyltrypt-amine derivatives: novel antagonists for the vascular 5-HT1B-like Receptor. J Med Chem 42:2504-2526

18. Oleson J, Sloboda A, Troy W, Halliday S, Landes M, Angier R et al (1955) The carcinostatic activity of some 2-amino-1,3,4-thiadiazoles. J Am Chem Soc 77:6713-6714

19. Krakoff $\mathrm{IH}$ (1964) Purine metabolism in the chick embryo: influence of 2-substituted thiadiazoles. Biochem Pharmacol 13:449-459

20. Oettgen HF, Reppert JA, Coley V, Burchenal JH (1960) Effects of nicotinamide and related compounds on the antileukemic activity of 2-amino-1,3,4-thiadiazole. Cancer Res 20:1597-1601

21. Shapiro DM, Shils ME, Fugmann RA, Friedland IM, Austin L, Clingen G et al (1957) Quantitative biochemical differences between tumor and host as a basis for cancer chemotherapy. IV Niacin and 2-ethylamino-1,3,4thiadiazole. Cancer Res 17:29-33

22. Hu Y, Li C, Wang X, Yang Y, Zhu H (2014) 1,3,4-Thiadiazole: synthesis, reactions, and applications in medicinal, agricultural, and materials chemistry. Chem Rev 114:5572-5610

23. Li P, Shi L, Yeng X, Yang L, Chen X, Wu F, Shi Q, Xu W, He M, Hu D, Song B (2014) Design, synthesis, and antibacterial activity against rice bacterial leaf blight and leaf streak of 2,5-substituted-1,3,4-oxadiazole/thiadiazole sulfone derivative. Bioorg Med Chem Lett 24:1677-1680

24. Dubey V, Pathak M, Bhat HR, Singh UP (2012) Design, facile synthesis, and antibacterial activity of hybrid 1,3,4-thiadiazole-1,3,5-triazine derivatives tethered via-S-bridge. Chem Biol Drug Des 80:598-604

25. Polkam N, Rayam P, Shree Anireddy J, Yennam S, Anantaraju H, Dharmarajan S, Perumal Y, Sravanti Kotapalli S, Ummanni R, Balasubramanian $S$ (2015) Synthesis, in vitro anticancer and antimycobacterial evaluation of new 5-(2,5-dimethoxyphenyl)-1,3,4-thiadiazole-2-amino derivatives. Bioorg Med Chem Lett 25:1398-1402

26. Supuran C, Scozzafava A (2000) Carbonic anhydrase inhibitors-part 94 1,3,4-Thiadiazole-2-sulfonamide derivatives as antitumor agents. Eur J Med Chem 35:867-874

27. Yusuf M, Khan RA, Khan M, Ahmed B (2013) An interactive human carbonic anhydrase-II (hCA-II) receptor-pharmacophore molecular model \& anti-convulsant activity of the designed and synthesized 5-amino-1,3,4-thiadiazole-2-thiol conjugated imine derivatives. Chem Biol Drug Des 81:666

28. Chapleo CB, Myers M, Myers PL, Saville JF, Smith AC, Stillings MR, Tulloch IF, Walter DS, Welbourn AP (1986) Substituted 1,3,4-thiadiazoles with anticonvulsant activity. 1. Hydrazines. J Med Chem 29:2273

29. Foroumadi A, Pournourmohammadi S, Soltani F, Asgharian-Rezaee M, Dabiri S, Kharazmi A, Shafiee A (2005) Synthesis and in vitro leishmanicidal activity of 2-(5-nitro-2-furyl) and 2-(5-nitro-2-thienyl)-5-substituted-1,3,4-thiadiazoles. Bioorg Med Chem Lett 15:1983

30. Yang X, Xiang L, Li X, Zhao T, Zhang H, Zhou W, Wang X, Gong H, Zu H (2012) Synthesis, biological evaluation, and molecular docking studies of 1,3,4-thiadiazol-2-amide derivatives as novel anticancer agents. Bioorg Med Chem 20:2789

31. Siddiqui N, Andalip N, Bawa S, Ali R, Afzal O, Akhtar M, Azad B, Kumar $R$ (2011) Antidepressant potential of nitrogen-containing heterocyclic moieties: an updated review. J Pharm BioAllied Sci 3(2):194-212

32. Rostom S, El-Ashmawy I, El Razik H, Badr M, Ashour H (2009) Design and synthesis of some thiazolyl and thiadiazolyl derivatives of antipyrine as potential non-acidic anti-inflammatory, analgesic and antimicrobial agents. Bioorg Med Chem 17:882-895

33. Chidananda N, Poojary B, Sumangala V, Kumari N, Shetty P, Arulmoli T (2012) Facile synthesis, characterization and pharmacological activities of 3,6-disubstituted 1,2,4-triazolo[3,4-b][1,3,4]thiadiazoles and 5,6-dihydro3,6-disubstituted-1,2,4-triazolo[3,4-b][1,3,4]thiadiazoles. Eur J Med Chem 51:124-136

34. Oruç E, Rollas S, Kandemirli F, Shvets N, Dimoglo A (2004) 1,3,4-Thiadiazole derivatives. synthesis, structure elucidation, and structure-antituberculosis activity relationship investigation. J Med Chem 47:6760-6767

35. Dilipkumar U, Damoder RM, Blake EW (2018) A unified strategy for the synthesis of $\beta$-carbolines, $\gamma$-carbolines, and other fused azaheteroaromatics under mild, metal-free conditions. Org Lett 20:6336-6339

36. Damoder RM, Dilipkumar U, Blake EW (2018) A general method for the metal-free, regioselective, remote $\mathrm{C}-\mathrm{H}$ halogenation of 8 -substituted quinolones. Chem Sci 9:1782-1788

37. Damoder RM, Frank RF, Blake EW (2016) Rh-catalyzed, regioselective, $\mathrm{C}-\mathrm{H}$ bond functionalization: access to quinoline-branched amines and dimers. Org Lett 18:5620-5623

38. Taha M, Shah SAA, Khan A, Arshad F, Ismail NH, Afifi M, Imran S, Choudhary MI (2015) Synthesis of 3,4,5-trihydroxybenzohydrazone and evaluation of their urease inhibition potential. Arabian J Chem. https://doi. org/10.1016/j.arabjc.2015.06.036

39. Imran S, Taha M, Ismail NH, Kashif SM, Rahim F, Jamil W, Wahab H, Khan KM (2015) Synthesis, in vitro and docking studies of new flavone ethers as a-glucosidase inhibitors. Chem Biol Drug Des 87(3):361-373

40. Imran S, Taha M, Ismail NH, Kashif SM, Rahim F, Jamil W, Hariono M, Yusuf M, Wahab H (2015) Synthesis of novel flavone hydrazones: in vitro evaluation of a-glucosidase inhibition, QSAR analysis and docking studies. Eur J Med Chem 105:156-170

41. Imran S, Taha M, Ismail NH, Fayyaz S, Khan KM, Choudhary MI (2015) Synthesis, biological evaluation, and docking studies of novel thiourea derivatives of bisindolylmethane as carbonic anhydrase II inhibitor. Bioorg Chem 62:83-93

42. Taha M, Ismail NH, Javaid K, Imran S, Wadood A, Ali M, Khan KM, Saad SM, Rahim F, Choudhary MI (2015) Evaluation of 2-indolcarbohydrazones as potent a-glucosidase inhibitors, in silico studies and DFT based stereochemical predictions. Bioorg Chem 63:24-35

43. Taha M, Ismail NH, Khan A, Shah SAA, Anwar A, Halim SA, Fatmi MQ, Imran S, Rahim F, Khan KM (2015) Synthesis of novel derivatives of oxindole, their urease inhibition and molecular docking studies. Bioorg Med Chem Lett 5:3285-3289

44. Morris GM, Huey R, Lindstrom W, Sanner MF, Belew RK, Goodsell DS, Olson AJ (2009) AutoDock4 and AutoDockTools4: automated docking with selective receptor flexibility. J Comput Chem 30(16):2785-2791

45. Wu L, Jiang J, Jin Y, Kallemeijn WW, Kuo CL, Artola M, Dai W, van Elk C, van Eijk M, van der Marel GA, Codee JDC, Florea BI, Aerts J, Overkleeft HS, Davies GJ (2017) Activity-based probes for functional interrogation of retaining $\beta$-glucuronidases. Nat Chem Biol 13:867-873 